\title{
Internal avalanches in models of granular media
}

\author{
Supriya Krishnamurthy ${ }^{a}$, Hans Herrmann ${ }^{a, b}$ Vittorio Loreto $^{a}$, Mario Nicodemi ${ }^{c}$ and Stephane Roux ${ }^{d}$ \\ a) P.M.M.H. Ecole Supérieure de Physique et Chimie Industrielles, \\ 10, rue Vauquelin, 75231 Paris CEDEX 05 France \\ b) ICA1, Univ. Stuttgart, Germany \\ c) Dipartimento di Fisica, Universitá di Napoli "Federico II", Unità INFM and INFN Napoli \\ Mostra d'Oltremare, Pad. 19, 80125 Napoli, Italy, \\ d) Laboratoire Surface du Verre et Interfaces, Unité Mixte de Recherche CNRS/St-Gobain, \\ 39, Quai Lucien Lefranc, F-93303 Aubervilliers Cedex, France.
}

\begin{abstract}
We study the phenomenon of internal avalanching within the context of recently introduced lattice models of granular media. The avalanche is produced by pulling out a grain at the base of the packing and studying how many grains have to rearrange before the packing is once more stable. We find that the avalanches are long-ranged, decaying as a power-law. We study the distriution of avalanches as a function of the density of the packing and find that the avalanche distribution is a very sensitive structural probe of the system.
\end{abstract}

\section{INTRODUCTION}

The internal structure and geometry of granular packings are very different from those of other systems such as liquids or solids and a lot of work has been devoted to understand them [1]. In particular, surface avalanches occurring in granular packings have been extensively studied [1]. In this paper we look at another sort of avalanching phenomenon also indicative of the internal structure - the phenomenon of internal avalanching occurring under small perturbations.

Recently simple lattice gas models have been proposed to describe slow dynamical processes in granular media, models whose basic ingredient is the geometric frustration in particle motion [2 [1]. These models reproduce experimentally observed phenomena such as slow relaxation in compaction, segregation [5], experimental irreversible-reversible cycles as well as the presence of "aging" and glassy behavior [6].

Within the context of these lattice gas models, we study in the present paper the features of internal avalanching. In particular we observe how the internal structure of the packing is reorganized after a small perturbation, such as pulling out a grain from the base. We find that surprisingly, the packing can undergo large rearrangements even under such a minimal perturbation. The size distribution of the produced events is very broad, being a power law over several orders of magnitude. This shows the strong sensitivity of packings, in the above models, to small perturbations which may trigger huge events up to the scale of the system itself. We study the avalanche distribution as a function of the density of the packing and find that the distribution shows a strong dependence on density, indicating that it is a very sensitive structural probe of the system.

The outline of the paper is as follows. In the following section, we define the model which we have studied numerically. In section $I I I$ we make precise the definition of an internal avalanche and define the quantity studied. Our results are described in section $I V$ and the conclusions and discussion follow in section $V$.

\section{TETRIS-LIKE MODELS}

In this section we briefly review the definitions and some basic properties of the Tetris-like lattice models [4] used in our simulations. The choice of the name "Tetris" captures the original idea of the computer game, of difficult parking problems in a packing of objects of different shapes. Frustration arises in granular packings owing to the interlocking of grains having different shapes. Different shaped particles have different sorts of excluded volume effects which leads to frustration in the packing. This geometrical feature is captured in this class of models. Hence, in this model, the complexity of the problem lies in the complexity of the particle arrangements in the packing. In a very general way we can define the model with a complex interaction matrix which tells us, for each particle, what are the constraints on the combinations of particles around it. For the sake of simplicity, in what follows, we define and study the model in its simplest version with two kinds of elongated particles.

In order to describe various experimentally observed properties of granular media, another class of frustrated lattice gas models with quenched disorder has also been introduced [2.37. Although in this paper we just focus on the Tetrislike models, it would be interesting to check the same phenomenology in this other class of frustrated models as well. 
The simplest version of the Tetris-like model can be defined by considering a system of particles which occupy the sites of a square lattice tilted by $45^{0}$ (see Fig. 1), with periodic boundary conditions in the horizontal direction (cylindrical geometry) and a rigid wall at the bottom. Particles cannot overlap and this condition produces very strong constraints (frustration) on their relative positions. For instance in the simplest case of two kinds of elongated particles pointing in two (orthogonal) directions, the frustration implies that two identical particles (pointing in the same direction) cannot occupy neighboring sites in this direction (Fig. 11). There is no other form of interaction between particles, and in this sense the model is purely geometrical. The system is initialized by filling the container, by inserting the grains at the top of the system, one at a time, and letting them fall under gravity. The particles perform an oriented random walk on the lattice until they reach a stable position defined as a position in which they cannot fall any further because of other particles below them.

The density reached by this filling procedure is $\sim 0.747$ and is the lowest density a random packing can reach in this model with two kinds of elongated particles (the density of a packing is measured by averaging over the densities of each row in the lower half of the system). Higher densities are reached by "shaking" the system, a procedure described below. This procedure has been studied earlier in relation to the experimentally observed slow density increase which occurs in these models 泊 as the shaking continues. In this paper however we concern ourselves with probing the changes in the structure of the packing caused by the shaking procedure, by studying internal avalanching as a function of the density.

The effect of vibrations is introduced by the possibility of the particles moving up with a probability $p_{u p}$ and of moving down with a probability $p_{\text {down }}=1-p_{\text {up }}$ The quantity $1 / \ln \frac{1}{x}$, with $x=p_{\text {up }} / p_{\text {down }}$, plays the role of an effective temperature and can be related to the tap intensity amplitude. The shaking procedure we use can be divided into two alternating steps. First, in a heating process (tapping) the system is perturbed by putting $p_{u p} \neq 0$ and performing a fixed number $N$ of attempts of movements per particle. At the end of this step, due to a nonzero $p_{u p}$, the system is in an unstable state with many particles in positions unsupported by particles below. We now allow the system to relax under gravity setting $p_{u p}=0$. The relaxation process (Cooling) is terminated when all the particles once more acquire stable positions. The system is now in a static state and the process of heating and relaxing the system is repeated a specified number of times. The density the system reaches depends, on average, on this number. The basic features of our model are robust with respect to variations in the exact Monte-Carlo procedure used.

This simple version of the Tetris model presents a trivial "antiferromagnetic" ground state. That is, the highest density packing that the system can reach with the above tapping procedure always has $\rho=1$ and is ordered in one of two possible orderings : Even(odd) rows consist of rods with $+45^{0}\left(-45^{0}\right)$ orientations. A state with only one kind of ordering is hence called a "single-domain" state. The existing of only two distinct orderings is potentially a drawback since a real granular system contains much more disorder due to a wider shape distribution and the absence of a lattice. In order to incorporate this effect, the Tetris model can be modified by considering particles with more complicated shapes [田珂]. This prevents the occurrence of an ordered ground state. In this paper however we study exclusively the simpler model described, with rods of two different orientations, with a brief discussion of the more complicated case in section $V$.

\section{INTERNAL AVALANCHES}

We study the effects of small perturbations on packings as a function of density, by studying in detail the phenomenon of internal avalanching within the scope of the models described above. Specifically we focus our attention on the rearrangements of grains generated in a static assembly by the extraction of a grain at the base. The creation of a void in the lattice destabilizes the neighboring grains above it. One of these may then fall down to fill the void, if the geometry of the packing allows the motion (i.e., if the orientation of the grain fits the local conformation). In this case, the net effect is that the void propagates one lattice step upwards destabilizing its neighbors in the layer above and so on. How effective this process is in causing the restructuring of the configuration depends on the precise structure of the packing. That large scale restructuring events are indeed possible is reflected in the fact that there are certain local configurations in which the motion of a single particle results in the motion of two particles above it, thus creating a second moving void. As a result of this "birth" process, moving voids can not only propagate up or get trapped but also multiply and hence lead to large avalanches.

We begin by preparing the system in the loose packed state by the procedure described in the previous section. As mentioned earlier, in this way the system attains in average a density of about $\rho=0.747\left(\rho_{l d}\right)$. The avalanche distribution in this state is studied by the following procedure. An initial state at the loose packed density is produced and a particle is randomly removed from the base. The total number of grains that move as a result of this removal is then calculated by checking the system row by row and letting all unstable particles settle under gravity. We have also checked that choosing unstable particles completely stochastically rather than row by row does not change the results 
quantitatively. By invoking the rules of stability introduced in this model, it is easy to see that when a particle is removed from the packing, it at most destabilizes all the particles within a cone with its apex at the removed particle (Fig. 11). Statistics for the avalanches is obtained by repeating this process of counting the number of unstable grains for various other initial states at the loose packed density. Thus the avalanche distribution obtained from this ensemble averaged procedure is indicative of this particular density. To obtain the distribution at higher densities the same ensemble averaging procedure is followed where now each member of the ensemble is generated by starting from an initial state at the loose packed density followed by a specified number of shakes. For each member of the ensemble, the magnitude of the internal avalanche is studied by counting as before the total number of particles rearranged as a result of removing one particle from the last row. The density that parametrises this avalanche distribution is just the average density of the ensemble. In everything that follows unless otherwise mentioned, this is the procedure we used to generate avalanche distributions at different densities, by averaging over statistics obtained for 100,000 different initial conditions. A figure of an actual avalanche is shown in Fig 2. The shaded dots represent the original positions of particles which moved as a result of the avalanche. The total number of these dots is then the quantity we measure as the size of the avalanche.

\section{RESULTS}

In studying the avalanche distribution as a function of the tapping density, we used two different values of $p_{u p}$ ( $p_{u p}=0.1$ and $\left.p_{u p}=0.5\right)$ in order to test the sensitivity of the results to different procedures. The avalanche size distribution for the loose-packed density is shown in Figure 3 as a function of the size of the system. As can be seen, it follows a power-law $P(s) \sim s^{-\tau}$ with an avalanche exponent $\tau$ close to 1.5. The distribution for the higher densities, according to the tapping procedure with $p_{u p}=0.1$ and $p_{u p}=0.5$, are shown in Figures 4 and 6 for a single system size $\left(L_{x}=200, L_{y}=200\right)$.

The density dependence of the avalanches is highly non-trivial. Since the loose packed density is the lowest that the packing can reach, shaking, as mentioned earlier, results in a monotonic increase of the density. It seems evident from Figure 3 and Figures 1 and 6 that the loose-packed density and other densities close to this value seem to exhibit a power-law behavior for the avalanche size distribution. At very high densities, when the structure is ordered (due to the ground state being a completely ordered one) the avalanche distribution is exponentially distributed (as will be clear later). Though this information is insufficient to infer the behaviour of intermediate densities, a possible hypothesis is that there exists a second order critical point located at some density $\rho_{c}$. Then one would expect that for densities larger than the critical one the system develops a characteristic size for the avalanches that acts as a cut-off for the avalanche size distribution. From this point of view one would then expect to be able to rescale all the avalanche size distributions obtained at different densities in one single scaling function such as

$$
P(s, \rho)=s^{-\tau} F\left(s\left(\rho-\rho_{c}\right)^{1 / \sigma}\right)
$$

where $\rho_{c}$ represents the location of the critical point and $P(s, \rho)$ is the probability for avalanches propagating in a medium of density $\rho$.

If we make this hypothesis of a single critical density even for the data, then the results for scaling the avalanche data in Figures (4) and (6) are shown in Figures 5 and 7 respectively. In each case, it is only the last three curves of the avalanche data that are scaled since the value of the critical density lies in between the lowest and the highest we have measured. As indicated the best value of the exponents $\tau$ and $\sigma$ seem to match for the two sets of data within the error bars. As for the values of $\rho_{c}$, though they seem to depend on the particular procedure used to generate the avalanches, we cannot rule out the possibility that they coincide within the error bar of our numerics.

In order to test this hypothesis of a single critical density in a simpler situation as well as elucidate the possible meaning of a critical density, we have also looked at a toy model which is a simple limiting case of the more general situation. As already mentioned, the Tetris model with rods of two orientations has a very simple ground state (highest density state) - the completely antiferromagnetic one. We take advantage of this fact by constructing the toy model in the following way. We begin with a $\rho=1$ completely antiferromagnetic state and generate lower density states by randomly removing particles. After each removal, the system is allowed to re-settle into a stable state via the avalanche dynamics already described. As a further simplification, we consider periodic boundary conditions in both $X$ and $Y$ directions. This allows us to eliminate system size effects as well as edge effects on the avalanche statistics. We call this example the Fully Periodic Single Domain (FPSD) model.

On this simplified version of the model, we perform the same set of measurements described earlier, in order to measure the avalanche distribution as a function of the density. A given density here is accessed by the removal of a certain number of particles instead of by shaking. Once a given density is reached, the avalanche distribution is measured by randomly removing a particle from the system and counting the number of particles destabilized as a 
result. A distribution is obtained by the same procedure of averaging over a ensemble of systems each originating from a $\rho=1$ state from which a given number of particles (corresponding to the density we want to access) is removed. The numerical results for avalanche size distribution as a function of the density is shown in Fig. 8. In this case the scaling hypothesis is satisfied and all the curves collapse for a critical density $\rho_{c}^{S D} \sim 0.76 \pm 0.01$, with an avalanche distribution decaying as a power with the exponent $\tau^{\prime}=1.45 \pm .05$ and $1 / \sigma=1.5 \pm 0.1$ (see Fig. (99)). As can be seen, the values of the scaling exponents are similar to those obtained for the original data.

The rationale for the existence of a critical point can be understood by mapping the avalanche to a problem of a branching process. The avalanche seed represents the insertion of a vacancy in the system and the subsequent evolution of the avalanche is just the propagation of this vacancy. There is an effective probability $p_{1}(\rho)$ for the vacancy to move through the medium, a probability $p_{0}(\rho)$ for stopping and a probability $p_{2}(\rho)$ for branching, i.e. meeting some other frozen vacancy and freeing it. In this mean field description, the critical point lies at the density which satisfies the condition $p_{2}(\rho)=p_{0}(\rho)$. In the FPSD case considered this mapping can be made quantitative by identifying the configurations leading to branching and death. It is possible to have in this way a mean-field estimate of the critical density, whose value is in good agreement with the numerical data presented in Fig. (9).

The FPSD does not fully reflect the complexity of the original problem since we basically probe an ordered state here while the configurations ensuing from shaking the loose-packed density (used to generate Figures 1 and 6) are disordered. The value of the critical density in the FPSD signifies the ordered loosest packing that a single domain state with periodic boundary conditions can achieve. The significance of a critical density in the "shaking" case is not so clear. The data seems to suggest a density larger than the loose packed value. However as mentioned earlier, we are unable to conclusively pick out a critical density in the latter case due to the large error bars.

It is obvious from Fig. (9) that the quality of the collapse is much better than in the case with the open boundaries. The error bars on the various parameters of the collapse are also reduced. This seems to imply that boundary conditions and surface effects play a dominant role in affecting avalanches. One reason that they might do this is the following. In the FPSD case, by virtue of the boundary conditions, the system is homogeneous and more or less has the same density everywhere. Whereas in the curves generated by shaking, the system develops a density profile with an interfacial region towards the top where the density decays to zero. This affects avalanche statistics by enhancing avalanches over a certain size. Avalanches which reach the low density region towards the top can very quickly move right to the surface. The width of the density profile can be reduced by tapping very gently, i.e. shaking with a very small amplitude. This is possibly an important point to be considered and taken into account when doing real life experiments in the laboratory to study internal avalanching.

\section{CONCLUSIONS AND DISCUSSION}

Our main conclusions are the following. The avalanche distribution is very sensitive to the internal structure of the packing and clearly appears to be long-ranged and decaying as a power law for a range of densities. As obvious in Figs. (4) and ( 6) a slight change in the density changes the avalanche distribution considerably. However as evident from the figures, the avalanche size cut-off is dominated by the system size over a range of densities, indicating that they are all effectively critical for the system sizes measured. Scaling behavior is thus visible only for the largest densities we have studied. The cleanest case, the case of the FPSD toy model seems to indicate that there is a critical density about which other densities obey the scaling relation Eq. 1 (Figs. 8 and 9). This might indicate one of two possibilities. The first is that there is a unique critical density $\rho_{c} \geq \rho_{l d}$ even in the case of Figs. 6 and 6 and we have to go to systems large enough that the density dependent cut off appears clearly in the avalanche distribution. A second possibility is that the avalanche distribution and hence the critical density crucially depends on the initial state begun with as well as the procedure under which the system reaches higher densities (as for example the case considered here, the case of different values of $\left.p_{u p}\right)$. Our numerical data thus far is unable to distinguish conclusively between these two possibilities.

In order to understand the role that the dynamics under which the system evolves plays in determining the internal structure of the medium (and hence the avalanche distribution) we have also studied the following dynamics as an alternative to shaking the system. We begin as usual with the loose packed density and take out a particle from the bottom row, hence initiating an avalanche. After the avalanche terminates, we add the particle back to the system at a random position on the top. This dual procedure of removing a particle and adding it back randomly to the top is continued till the system reaches a steady state in which the avalanche distribution is studied. We find that in this case, the steady state the system reaches is different from any reached in the packing procedure in that the avalanche distribution decays with a different power $\tau=1.75 \pm 0.05$. Further details of this procedure as well as a description of the steady state reached are reported elsewhere [7].

There are several possibilities which remain to be investigated. Amongst the most important of these is how much 
of this scenario holds for similar models with particles of more complicated shapes. An example is the Tetris-like model with "T"-shaped particles [., F. As mentioned earlier, this model has a highly degenerate ground state unlike the simpler model we have studied in this paper. It is of interest to investigate whether this property affects the scenario discussed above for the avalanche distribution as a function of density. While preliminary results indicate that the avalanche distribution is again a power-law in this case, we have yet to investigate in detail its density or dynamics dependence.

\section{ACKNOWLEDGEMENTS}

We would like to thank S. Zapperi for useful comments. We are grateful to CEFIPRA for their support. In particular SK would like to acknowledge financial support under project no 1508-3/192. VL acknowledges financial support under project ERBFMBICT961220. This work has been partially supported from the European Network-Fractals under contract No.FMRXCT980183. 
[1] for a general introduction to the overall phenomenology of granular matter one can address the following papers: H.M. Jaeger and S.R. Nagel, Science 255, 1523 (1992); H.M. Jaeger, S.R. Nagel and R.P. Behringer, Rev. Mod. Phys. 68, 1259 (1996). D. Bideau and A. Hansen, eds. Disorder and Granular Media, (North-Holland, Amsterdam, 1993). A. Mehta, ed., Granular Matter: an interdisciplinary approach, (Springer-Verlag, New York, 1994). See also Proceedings of the NATO Advanced Study Institute on Physics of Dry Granular Media, Eds. H. J. Herrmann et al, Kluwer Academic Publishers, Netherlands (1998).

[2] A. Coniglio and H.J. Herrmann, Physica A, 225, 1 (1996).

[3] M. Nicodemi, A. Coniglio and H.J. Herrmann, Phys. Rev. E 55, 3962 (1997); J. Phys. A 30, L379 (1997); Physica A 240, 405 (1997).

[4] E. Caglioti, V. Loreto, H.J. Herrmann and M. Nicodemi, Phys. Rev. Lett. 79, 1575 (1997) and in preparation (1998).

[5] E. Caglioti, A. Coniglio, H.J. Herrmann, V. Loreto and M. Nicodemi, Europhys. Lett. 43, 591 (1998).

[6] M. Nicodemi and A. Coniglio, preprint (1998), cond-mat/9803148.

[7] S. Krishnamurthy, H. J. Herrmann, V. Loreto and S. Roux, in preparation (1998). 
Figure Captions

Fig. 1 An example of a stable configuration on which internal avalanche measurements can be made. If the circled particle is removed, from the lower most layer, it at most destabilizes all the particles within the cone shown. The boundary conditions are periodic in the horizontal direction $(X)$.

Fig. 2 A picture of an avalanche. The shaded dots represent all the particles in the initial configuration which moved as a result of the removal of a particle from the lower most layer.

Fig. 3 Avalanche size probability distribution $P(s)$ for the loose-packed density for different system sizes. In increasing order, the system sizes are $L x=100, L y=100, L x=200, L y=200, L x=200, L y=400$ and $L x=100, L y=900$. Average over 100, 000 realizations.

Fig. $4 P(s)$ vs. $s$ for the densities mentioned for $p_{u p}=0.1$.

Fig. 5 The scaling plot $f\left(s^{*}\right)$ vs. $s^{*}$ of the data shown in Fig. 4 where $s^{*}=s\left(\rho-\rho_{c}\right)^{1 / \sigma}$ and $f\left(s^{*}\right)=\left(s^{*}\right)^{-\tau} F\left(s^{*}\right)$. The last three densities are scaled with parameters $\tau=1.5 \pm 0.1,1 / \sigma=1.5 \pm 0.1$ and $\rho_{c}=0.77 \pm 0.1$

Fig. $6 P(s)$ vs. $s$ for the densities $\rho=0.76-0.83$ for $p_{u p}=0.5$.

Fig. 7 The scaling plot $f\left(s^{*}\right)$ vs. $s^{*}$ of the data shown in Fig. 6 where $s^{*}=s\left(\rho-\rho_{c}\right)^{1 / \sigma}$ and $f\left(s^{*}\right)=\left(s^{*}\right)^{-\tau} F\left(s^{*}\right)$. The densities scaled are $\rho=0.81-0.83$ of the data shown in Figure 6. The scaling parameters are $\tau=1.5 \pm 0.1$, $1 / \sigma=1.5 \pm 0.1$ and $\rho_{c}=0.79 \pm 0.1$

Fig. $8 \quad P(s)$ vs. $s$ for the densities mentioned for the FPSD model.

Fig. 9 The scaling plot for the data in Fig. 8. with the scaling parameters $\tau=1.45 \pm 0.05,1 / \sigma=1.5 \pm 0.1$ and $\rho_{c}=0.76 \pm 0.01$ 


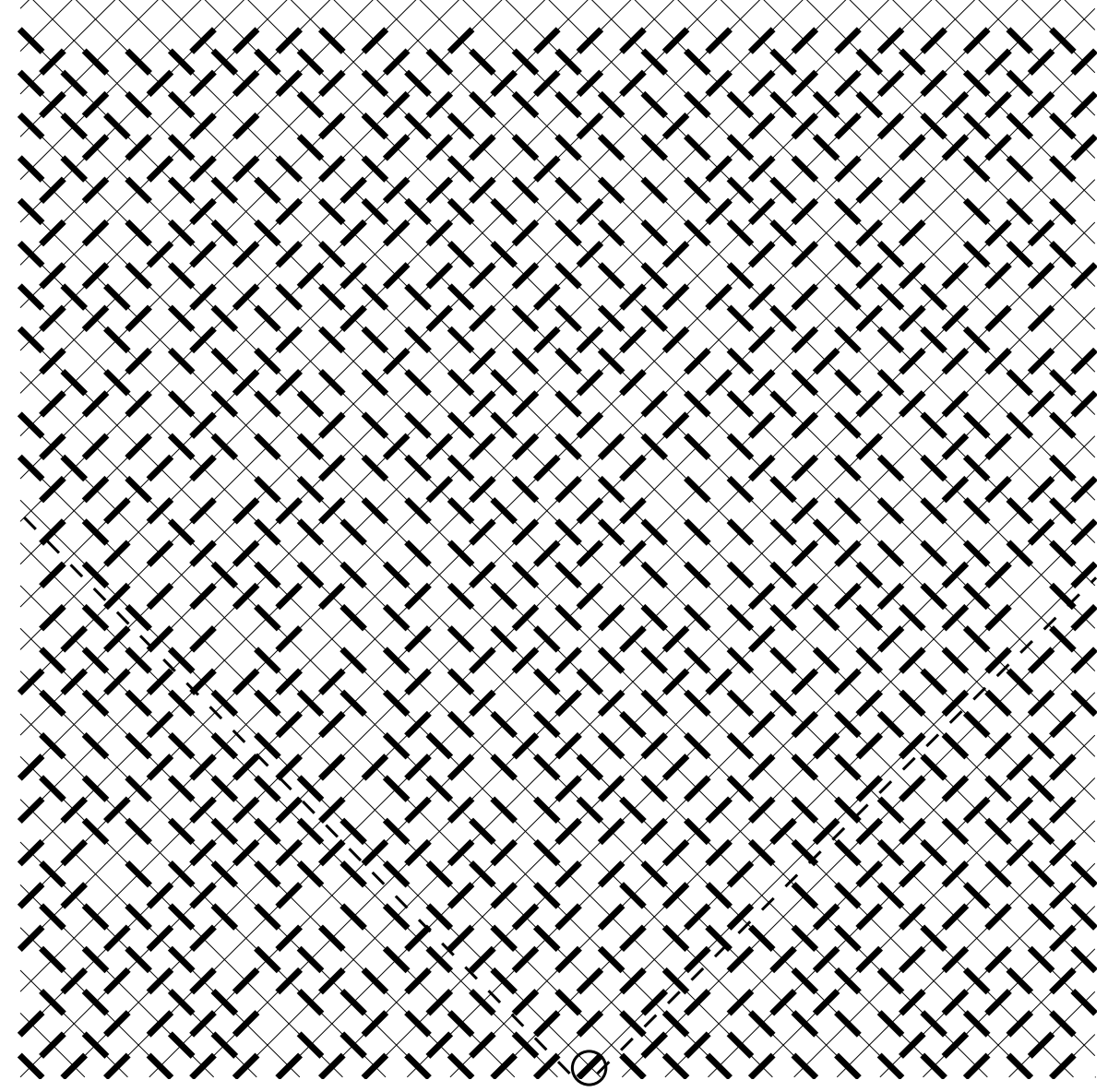

FIG. 1. 


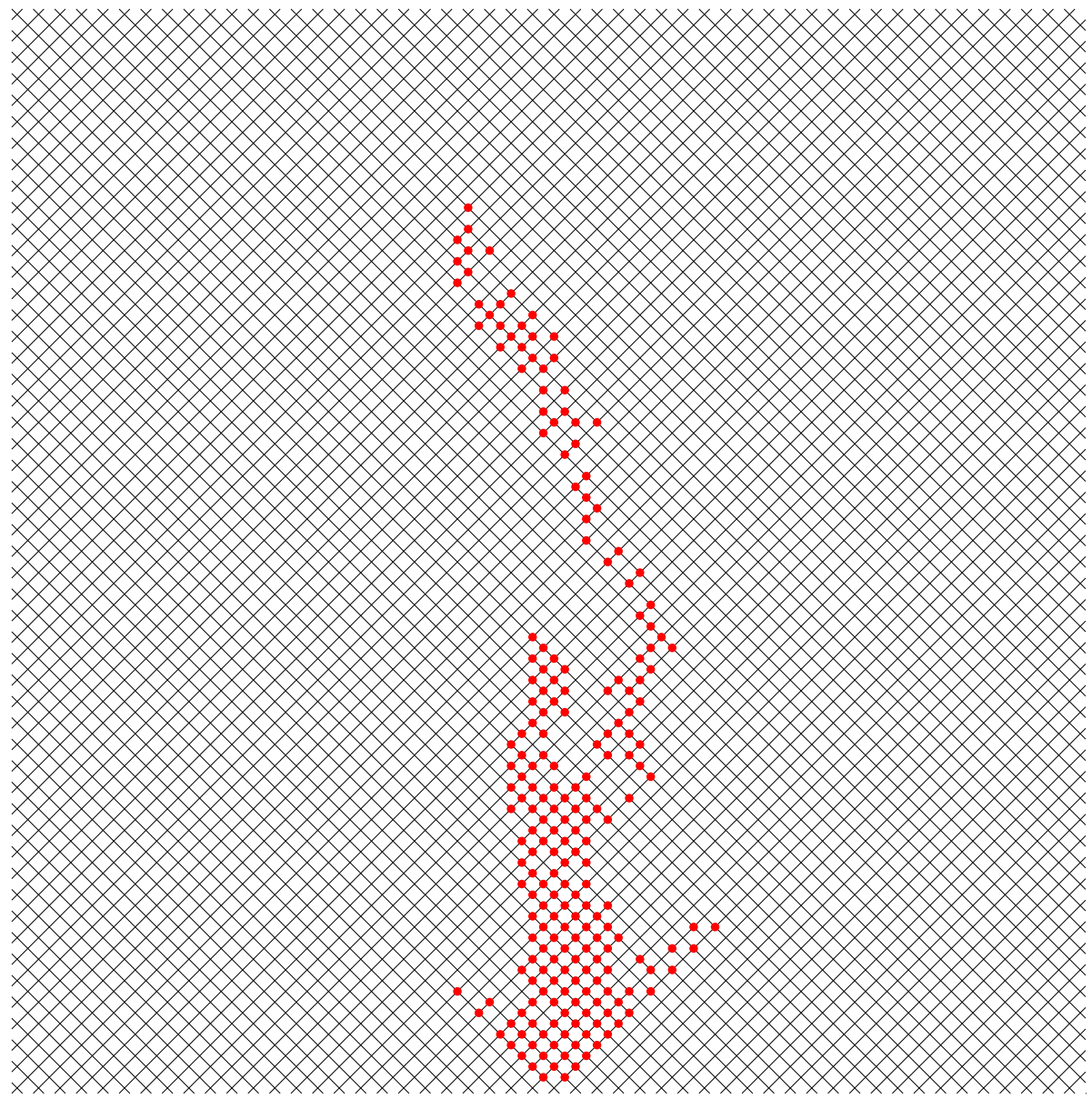

FIG. 2

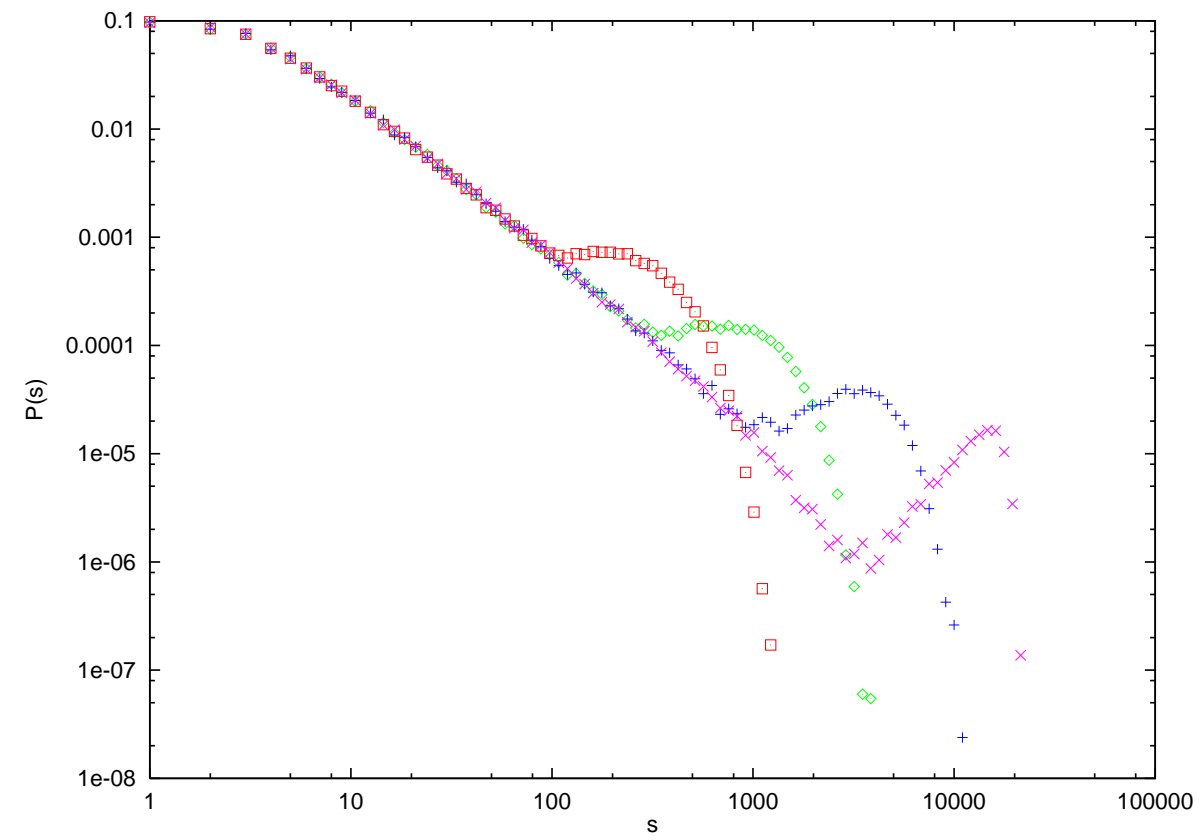

FIG. 3. 


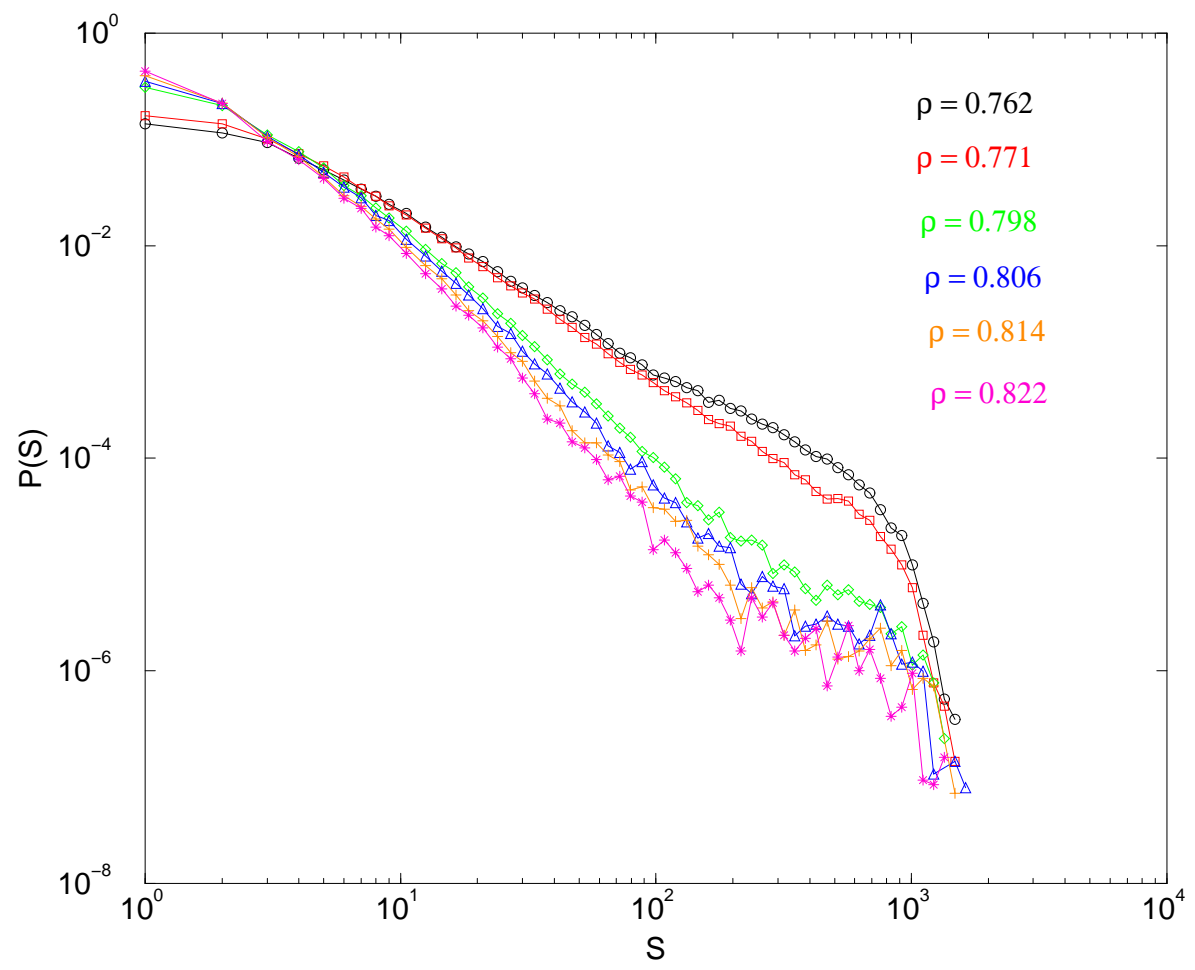

FIG. 4.

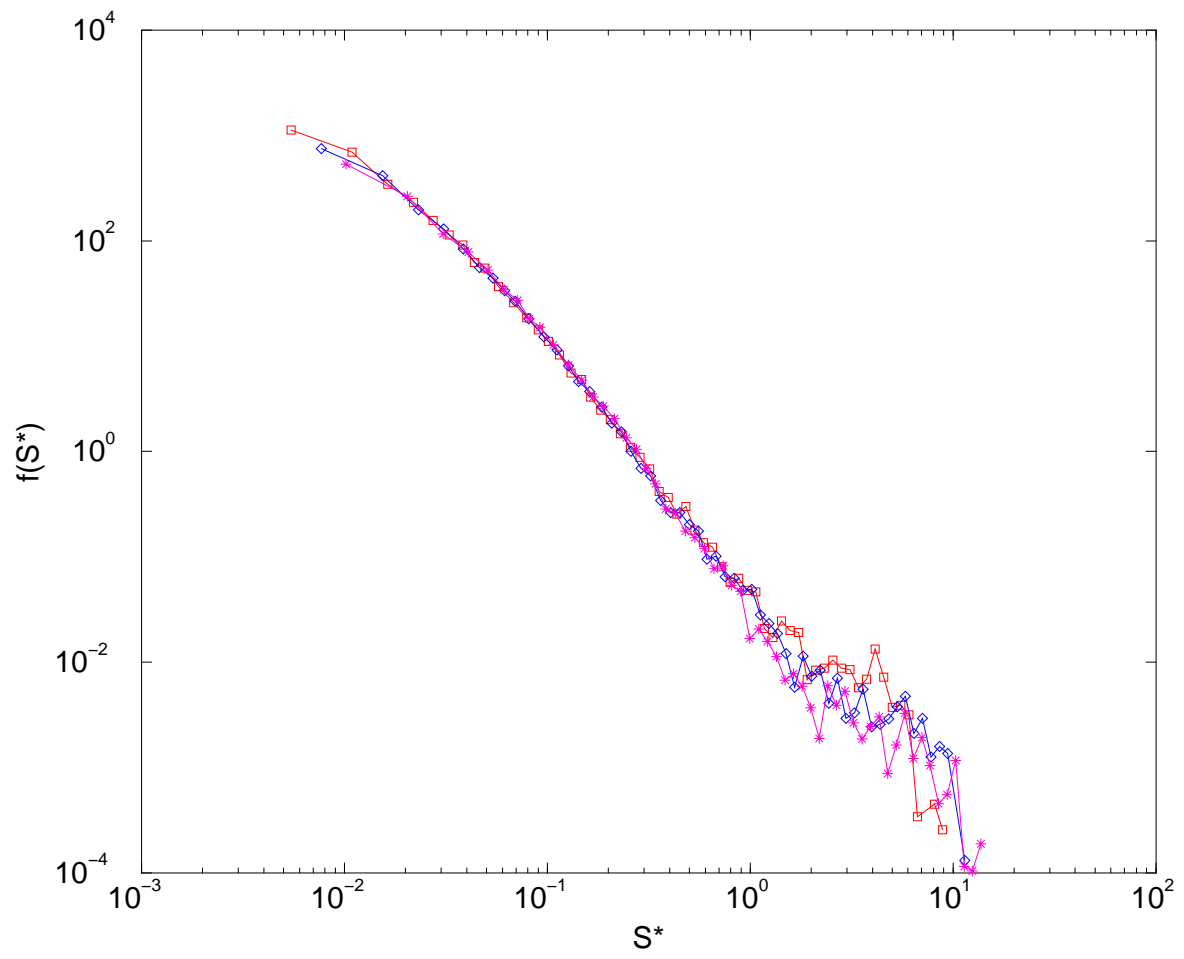

FIG. 5. 


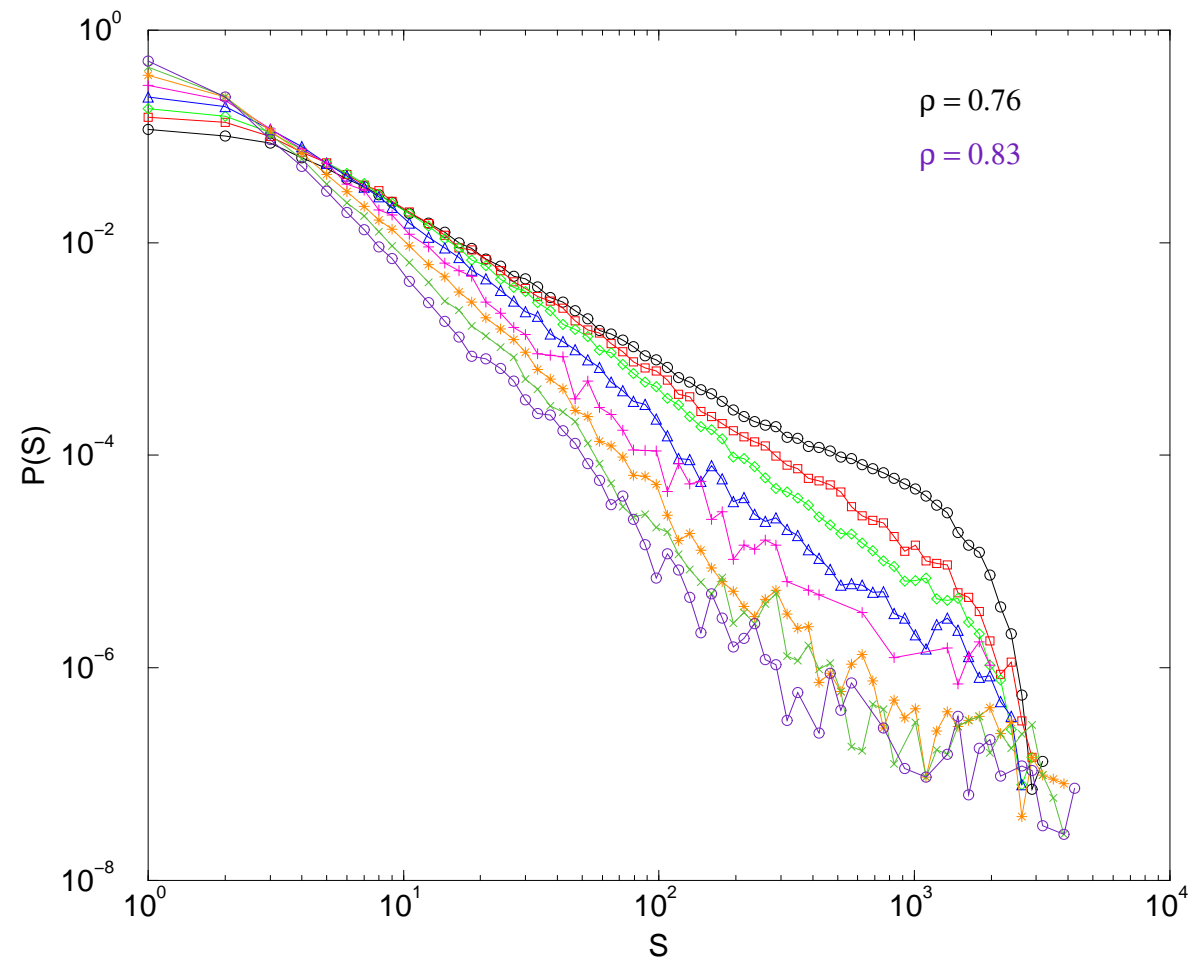

FIG. 6.

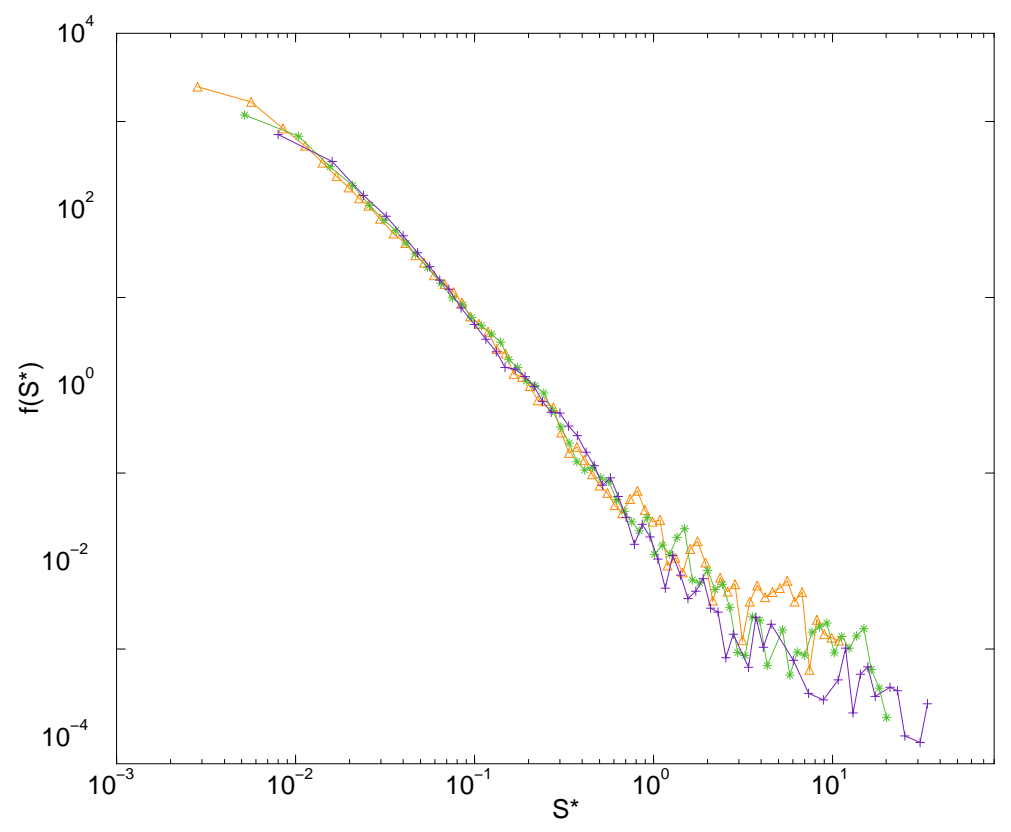

FIG. 7. 


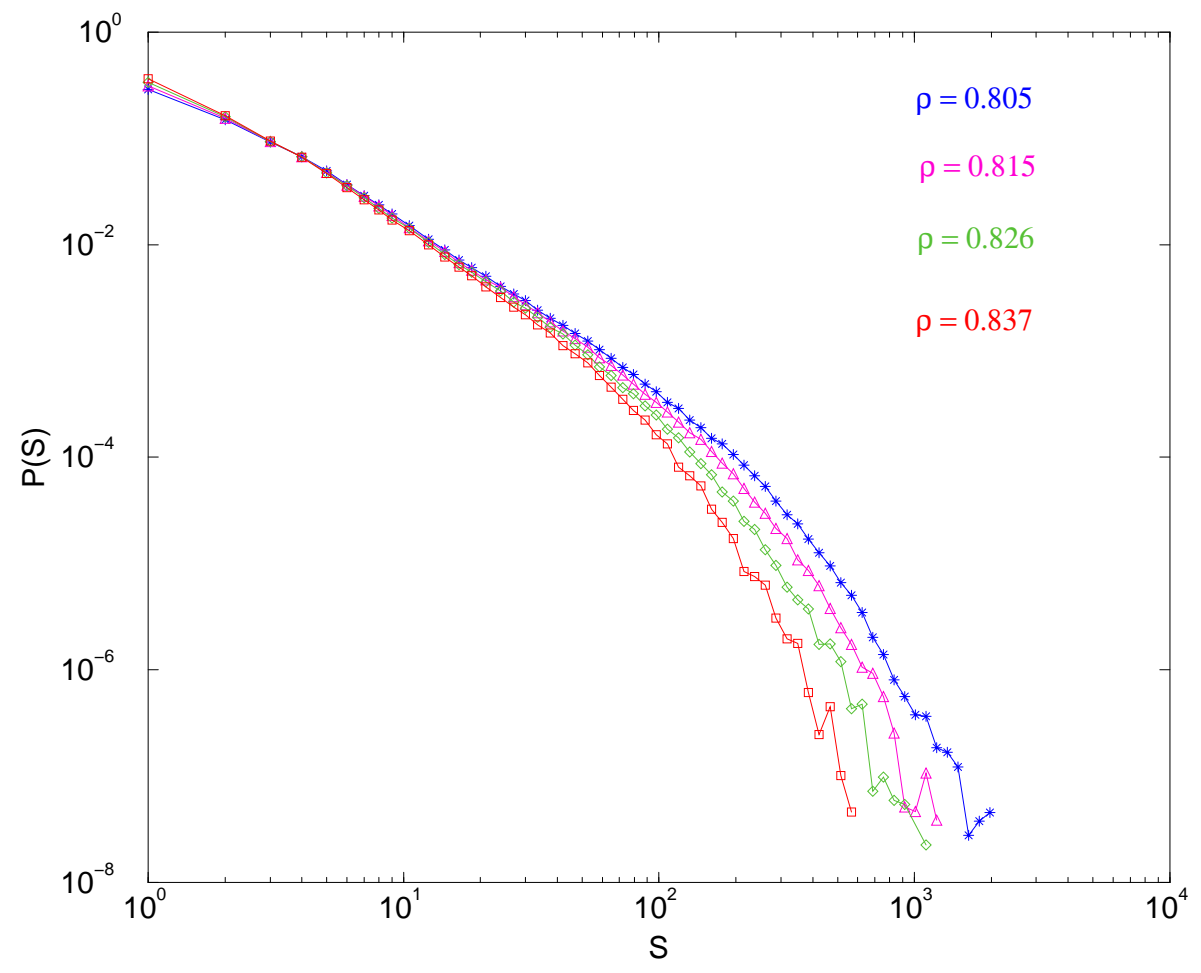

FIG. 8.

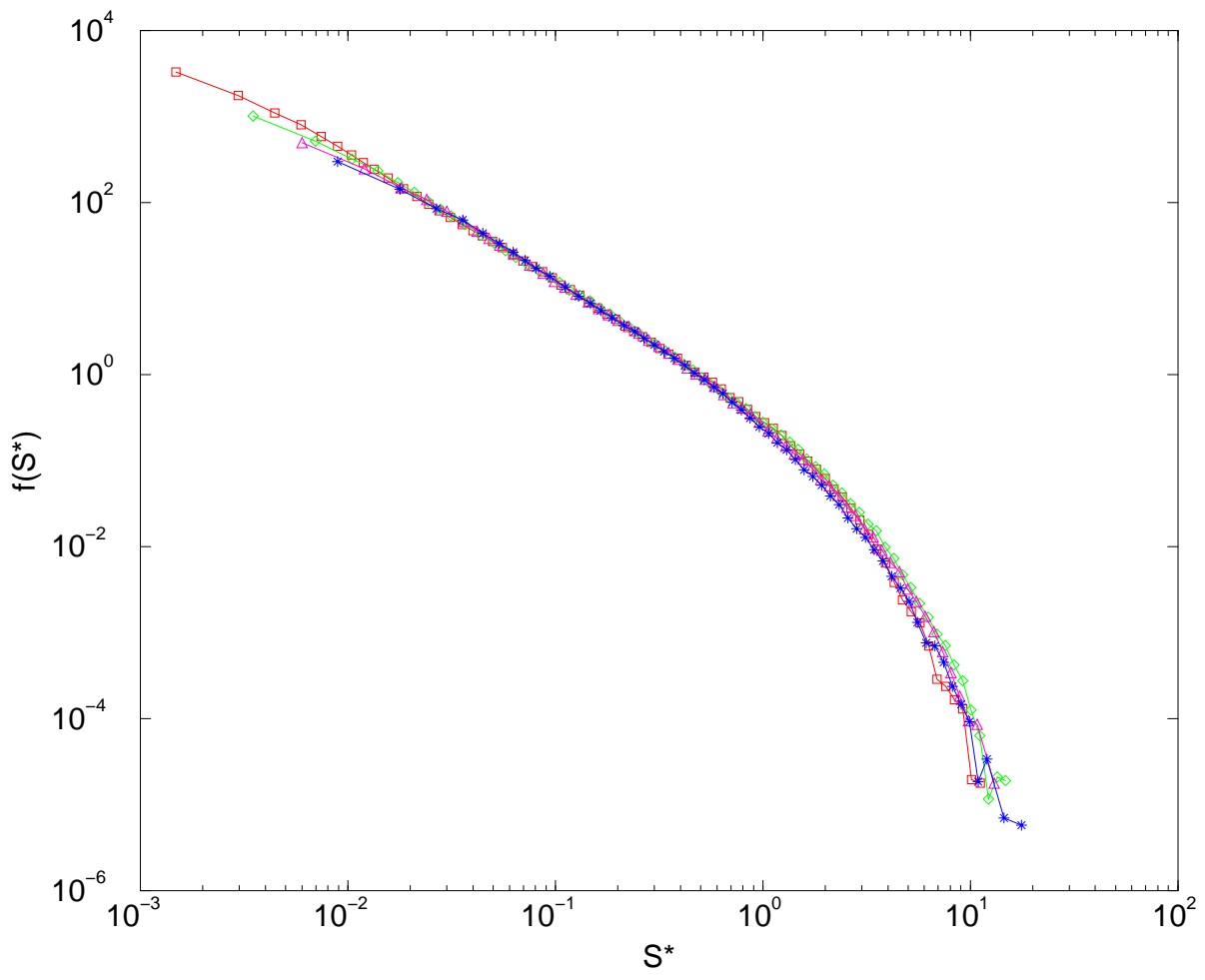

FIG. 9. 